\title{
Water Hammer Induced Cavitation - A Numerical and Experimental Study
}

\author{
Marcus Jansson, Magnus Andersson*, Maria Pettersson, and Matts Karlsson* \\ Atlas Copco Rock Drills AB, SE-701 91 Örebro, Sweden \\ E-mail: marcus.jansson@se.atlascopco.com, magnus.andersson@liu.se, maria.pettersson@se.atlascopco.com, \\ matts.karlsson@liu.se \\ *Division of Applied Thermodynamics and Fluid Mechanics, Department of Management and Engineering, Linköping \\ University, Sweden
}

\begin{abstract}
Cavitation erosion is one of the main concerns in hydraulic rock drills and can reduce both performance as well as life span. Current simulation tools can detect a potential risk of cavitation, however, the equations do not include cavitation physics and therefore cannot estimate the severity nor erosion locations. In order to evaluate the cavitation damage, long term tests are performed which are both costly and time consuming. With better computational capacity and more accurate numerical flow models, the possibilities to simulate the course of cavitation have increased. So far, most numerical studies on cavitation focus on steady-state problems while studies on hydraulic transients and water hammer effects have received less attention. This paper is a step towards simulation of water hammer induced cavitation and cavitation erosion in pipe flow using Computational Fluid Dynamics (CFD). In order to validate the results, experimental measurements are performed with a test equipment that creates hydraulic transients in a pipe and records these using piezoelectric pressure sensors. The results from CFD are compared to both the experimental data and to numerical results from a software called Hopsan, a one-dimensional multi-domain system simulation tool that uses wave characteristics to calculate pressures and flows. For smaller transients where no cavitation occur, all results show good agreement. For larger transients with cavitation, the results from Hopsan do not longer agree with the measurements, while the CFD model still performs well and is able to predict both formation and collapse of cavitation.
\end{abstract}

Keywords: Cavitation, Water hammer, Hydraulic transients, Rock drills, CFD

\section{Introduction}

Cavitation erosion is a major failure mechanism for hydraulic rock drills and is a cause for both reduced performance and reduced life span [1,2]. With the simulation tools that are currently used it is possible to detect situations where cavitation might occur, however, these tools do not include any cavitation physics and cannot predict the severity nor the erosion sites. Therefore, long term tests, which are both costly and time consuming, are performed in order to evaluate the cavitation erosion.

Cavitation can be described as the rapid growth, and collapse, of bubbles in a liquid due to large pressure gradients. A liquid at a pressure below the saturation vapor pressure is said to be under tension. If no vapor or gas is present in the liquid it can sustain a considerable amount of tension and still remain stable. In reality however, small imperfections such as microbubbles of non-condensable gases or small suspended solid particles act as nucleation sites. At a certain tension, the liquid will rupture and form cavitation bubbles [3]. The evaporation/condensation at the bubble surface is very fast com- pared to the bubble dynamics and the vapor pressure inside the bubble can be considered constant. The bubbles contain mostly vapor but also small amounts of gas, e.g. air, that are dissolved in the liquid. The evaporation/condensation time scale is much shorter than that of diffusion. Thus the amount of non-condensable gases in the bubble is almost constant and the partial pressure of these gases varies with the bubble radius. When the ambient pressure is increased, the bubbles will collapse. The vapor inside the bubble will condense, however, the dissolved gases will instead be highly compressed. The pressure inside the bubble will thus increase during the collapse and eventually exceed the ambient pressure, forcing the process to revert and the bubble to rebound. The collapse of a cavitation bubble is often followed by multiple rebounds and collapses. The bubble continues to rebound and collapse until the gases have had time to diffuse to the liquid. Each collapse emits a large amplitude pressure wave $[4,5]$. Bubbles collapsing close to a solid boundary tend to collapse more rapidly at the side facing away from the surface. This will form a liquid jet that punctures the bubble. This re-entrant jet can reach velocities of several hundreds of meters per second. 
The bubble will eventually split into parts and the jet will instead hit the wall surface. The impact pressure from either the jet or from the pressure wave can locally exceed both the yield limit and the ultimate strength of many materials. In this case, local damage is induced. Most metals have an incubation period when the damage is isolated to small zones of plastic deformation, so called pits. During this time, no measurable mass loss occurs. When the pits become numerous they start to overlap and when the surface has been exposed to sufficiently many of these superimposed impacts the material fails. The incubation period is followed by extensive material loss. The severity of the cavitation erosion is affected by both the exposure time and the cavitation intensity $[3,6]$.

The use of Computational Fluid Dynamics (CFD) for solving various problems regarding fluid flow has increased as the performance of the methods and the computational resources are improved. However, CFD is not that extensively used for hydraulic systems. Often, less computationally expensive methods are used to solve for pressure and flows such as the Methods of Characteristics [7]. These methods are in most situations both quick and robust, however they lack in solving some of the physics that may be encountered. More recent studies have shown the value of CFD when determining the effects of water hammer in pipes [8]. Further, CFD have been used successfully to model cavitation and the risk of cavitation erosion in diesel injectors $[9,10]$ and hydraulic valves $[11,12]$. However, the cavitation in these studies is a consequence of the accelerated flow and cavitation as the result of hydraulic transients has not been considered.

In addition to predict cavitation inception, the ultimate goal is to predict sites of cavitation erosion. Pressure [6], jet velocity [13] and the condensation source term in the vapor continuity equation [10] are all quantities that have shown potential for cavitation erosion prediction. This study aims to evaluate the possibility to use CFD as a tool for predicting cavitation formation and collapse caused by water hammer pulsations.

\section{Experimental setup}

The equipment consists of a pressurized pipe that is connecting two volumes (fig. 1), and a control valve that is located at the high pressure side of the pipe. With this valve it is possible to completely shut the flow in the pipe. At the low pressure side there are two volumes connected by an orifice. The orifice is exchangeable in order to vary the diameter and thus the flow rate. Dimensions are found in tab. 1.

Table 1: Dimensions for test equipment.

\begin{tabular}{ccc}
\hline & Length $[\mathrm{mm}]$ & Radius $[\mathrm{mm}]$ \\
\hline Tank 1 & 85 & 50 \\
Pipe & 755 & 5 \\
Tank 2 & 85 & 50 \\
Orifice & 1 & $0.5-2$ \\
Tank 3 & 95 & 50 \\
\hline
\end{tabular}

The system is in steady-state initially. The pressure in each

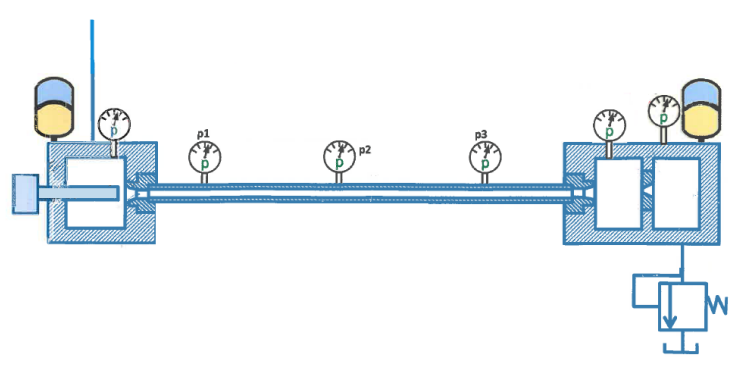

Figure 1: Simple representation of the test equipment

of the volumes are measured using silicon pressure sensors. The accuracy of these are $\pm 0.2 \%$ of the full scale. In the first and second tank, i.e. the high pressure side, the measurement range is $0-25 \mathrm{GPa}(0-250 \mathrm{bar})$. In the third tank, i.e. the low pressure side, the measurement range is $0-9 \mathrm{GPa}(0-9$ bar).

At a given time, the valve is closed rapidly. The exact movement of the valve is measured with a laser while another laser is recording any potential movement of the equipment itself.

The pressure pulsations in the pipe due to the valve closing are registered by three piezoelectric pressure sensors along the pipe which measures the pressure dynamically. These are located at a distance of $\mathrm{P} 1=100 \mathrm{~mm}, \mathrm{P} 2=380 \mathrm{~mm}$ and $\mathrm{P} 3=660$ $\mathrm{mm}$ from the pipe opening (fig. 1). The resolution of these sensors is $0.14 \mathrm{kPa}$ and the sampling rate is $1 \mathrm{MHz}$.

Tests with both $2 \mathrm{~mm}$ and $4 \mathrm{~mm}$ orifice diameters are performed. In both cases the inlet pressure is $6 \mathrm{GPa}$ (60 bar) and oil viscosity $0.04 \mathrm{~Pa} \cdot \mathrm{s}(40 \mathrm{cP})$. With a $2 \mathrm{~mm}$ orifice, the flow rate is $2.68 \times 10^{-4} \mathrm{~m}^{3} / \mathrm{s}(16.1 \mathrm{l} / \mathrm{min})$. The total stroke length of the valve is $2 \mathrm{~mm}$ and the closing time is $7.2 \mathrm{~ms}$. The overlap between valve and pipe is $1 \mathrm{~mm}$ and the valve reaches the pipe opening after $1.8 \mathrm{~ms}$. With a $4 \mathrm{~mm}$ orifice, the flow rate is $9.63 \times 10^{-4} \mathrm{~m}^{3} / \mathrm{s}(57.8 \mathrm{l} / \mathrm{min})$. The closing time is $2.7 \mathrm{~ms}$ and the valve reaches the pipe opening after $0.77 \mathrm{~ms}$.

\section{Numerical setup}

Two different numerical strategies are executed. The first is based on CFD where the Reynolds Averaged Navier-Stokes (RANS) equations are solved using the finite volume method. The second is a one-dimensional approach using the Hopsan software, which is a multi-domain system simulation tool that uses wave characteristics to calculate pressures and flows.

\subsection{Computational Fluid Dynamics}

The coupled pressure based solver in ANSYS Fluent 17.2 is used with the Realizable $k-\varepsilon$ turbulence model [14] and enhanced wall treatment. The medium is assumed to be a homogeneous mixture of oil and oil vapor. The oil is a compressible liquid while the vapor is treated as an incompressible gas. The mass transfer between phases is included using the Schnerr-Sauer cavitation model [15] based on the RayleighPlesset equation for bubble radius [4]. The mass transfer rate for evaporation and condensation can be seen in eq. (1) and eq. (2) respectively 


$$
\begin{aligned}
& R_{e}=\frac{\rho_{v} \rho_{l}}{\rho} \alpha(1-\alpha) \frac{3}{R_{B}} \sqrt{\frac{2}{3} \frac{\left(P_{v}-P\right)}{\rho_{l}}} \\
& R_{c}=\frac{\rho_{v} \rho_{l}}{\rho} \alpha(1-\alpha) \frac{3}{R_{B}} \sqrt{\frac{2}{3} \frac{\left(P-P_{v}\right)}{\rho_{l}}}
\end{aligned}
$$

where $R_{e}$ and $R_{c}$ are the mass transfer rates by evaporation and condensation. $\rho$ is the mixture density while $\rho_{v}$ and $\rho_{l}$ are the densities for vapor and liquid phases. $\alpha$ is the vapor volume fraction. $P_{v}$ is the vapor pressure and $P$ is the local far-field pressure. The bubble radius, $R_{B}$, is described by

$$
R_{B}=\left(\frac{\alpha}{1-\alpha} \frac{3}{4 \pi} \frac{1}{n}\right)^{\frac{1}{3}}
$$

where $n$ is the bubble number density.

The computational domain is assumed to be axi-symmetric and discretized by $413 \mathrm{k}$ quadrilateral cells. A fixed time step size of $1 \mu \mathrm{s}$ is utilized, which corresponds to a Courant number below 3 (speed of sound) or 1 (fluid velocity). Sensitivity analysis on both the spatial and temporal resolution were performed and showed no major result changes with further refinement.

\subsection{Hopsan}

The model, seen in fig. 2, is very simplified and does not contain any of the components that are used in order to control the valve. Instead, the valve movement is controlled by a table with time and positions. These values are obtained from laser measurements of the valve motion.

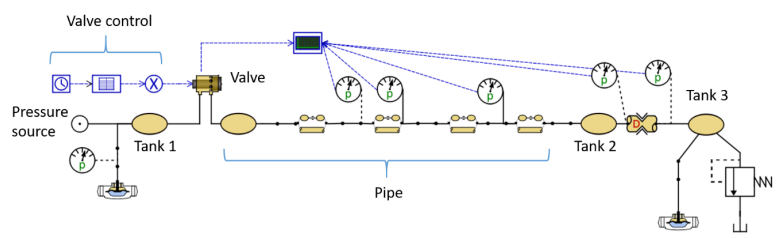

Figure 2: A simplified model of the test equipment that is used for the $1 D$ simulations in Hopsan.

The inlet is represented by a pressure source with a specified pressure. It is possible to specify the radial clearance of the spool valve in order to take the leaking into account. Furthermore it is possible to change the diameter of the orifice.

\section{Results and discussion}

Results from the experimental setup are limited to the transient pressure in the pipe. These pressure curves are compared to numerical results from both Hopsan and CFD. In Hopsan, three different values for the spool radial clearance are represented. Furthermore, some brief results from methods of cavitation erosion prediction are presented.

\subsection{Hydraulic transients}

When using a $4 \mathrm{~mm}$ orifice, the cavitation is severe (fig. 3). The CFD results show that the cavitation has a higher effect than might be expected. When the pressure wave is reflected against a cavitation cloud it behaves differently compared with reflection against the valve. The result is small pressure oscillations at relatively low pressure that in some sense interfere with one and other. When the cavitation cloud eventually collapses, a large amplitude, high pressure wave appears. This wave is reflected at the tank in accordance to water hammer theory and returns to the valve as a low pressure. Cavitation occur once again and the procedure is repeated. The resulting transient pressure profile looks nothing at all like classic water hammer theory. Instead, small pressure variations are followed by a single large pressure wave and so forth.
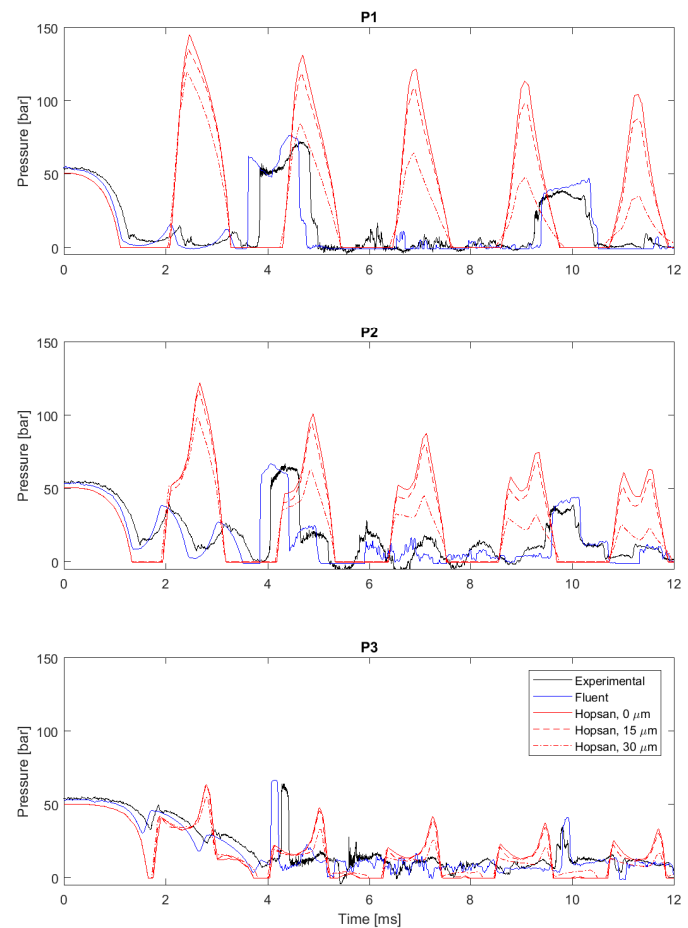

Figure 3: Experimental and numerical results for $4 \mathrm{~mm}$ orifice. Experimental data is presented in black and CFD (Fluent) results in blue. Hopsan results are shown in red with the different style lines representing various spool clearances.

A smaller orifice reduces the flow rate and thus the amplitude of the transients (fig. 4). The amplitude of the pressure wave is not sufficient to reduce the pressure to vapor pressure. The resulting pressure profile looks like what could be expected from water hammer. Furthermore, numerical results from Hopsan and CFD both agree fairly well with experiments. The types of damping in the different systems must 
be considered. In the CFD model, it is assumed that there is no leakage at all between the valve and the pipe wall. The damping mainly consists of inertial and viscous forces. The turbulent viscosity from the turbulence model contributes to the damping. In the experimental setup, there is some leakage that greatly contributes to the damping of hydraulic transients. The difference between CFD results and experiments is most likely due to the leaking valve. In Hopsan, different values for the radial spool clearance are defined. It can be seen that the damping of transients is highly affected by this clearance, and thus the amount of leakage.
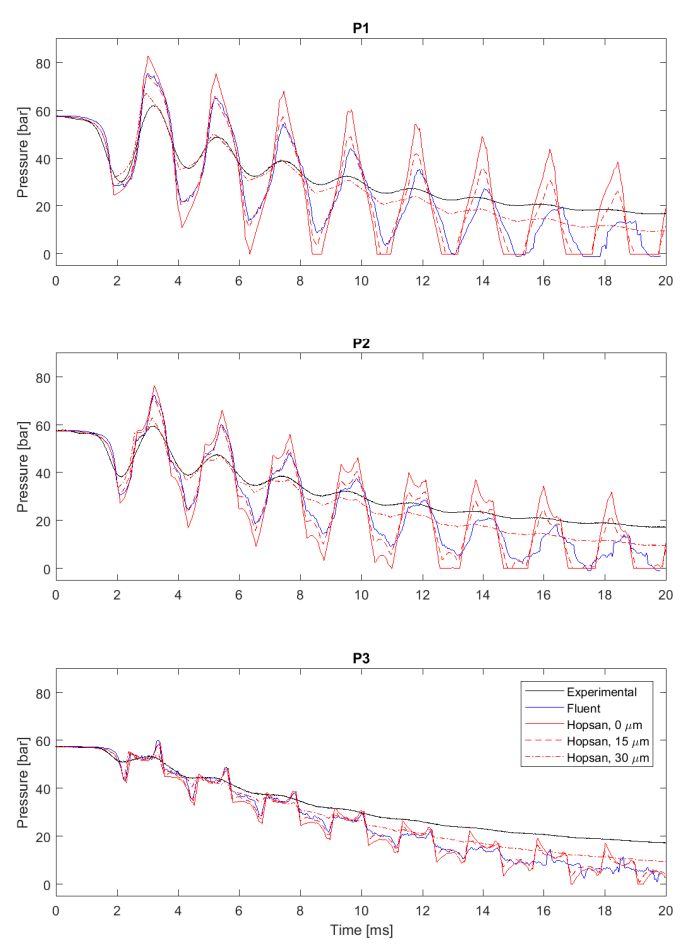

Figure 4: Experimental and numerical results for $2 \mathrm{~mm}$ orifice. Experimental data is presented in black and CFD (Fluent) results in blue. Hopsan results are shown in red with the different style lines representing various spool clearances.

\subsection{Cavitation erosion}

Prior to the moment of the first collapse (fig. 5), it is possible to detect two regions with increased wall condensation rate which might be more exposed to cavitation erosion. However, since this is just a snapshot, the transient effects of cavitation erosion cannot be determined.

The lowest wall pressure can be coupled to the regions in the pipe with vapor (fig. 6). This method cannot however distinguish between evaporation and condensation. The condensation phase should be characterized by large amplitude pressure waves from bubbles collapsing. This effect is not seen in the wall pressure.

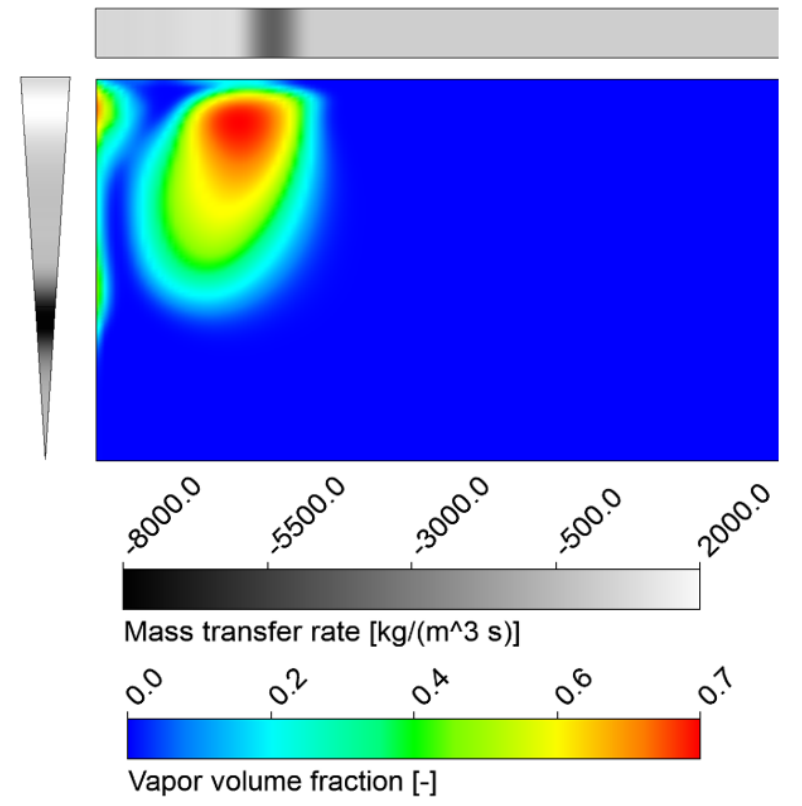

Figure 5: Vapor volume fraction inside the pipe and mass transfer rate at the pipe walls.

\subsection{Limitations and future work}

This study is only focused on accurately predict the pressure pulsations in a cavitating flow. The behaviour of the cavitation itself is currently not validated using any method. If the pipe in the test equipment would be of a transparent material it might be possible to detect cavitation optically and in that way validate if the cavitation bubbles are correctly modelled. If so, it could be possible to predict where the cavitation bubbles will end up and eventually collapse.

Methods for predicting the risk for cavitation erosion are not validated. However, CFD is considered a very promising tool and there are a number of different approaches that might be appropriate. The condensation source term in the vapor transport equation is a parameter that has great potential for transient flow. By using the time integral of this parameter it is possible to determine the amount of vapor that has condensated at e.g. a wall. By comparing this, or some other accumulative quantity, to experimental data of material loss it should be possible to tailor a model for prediction of cavitation erosion.

Numerical results are only compared to one set of experiments. For further studies, repeatability of the experiments should be considered and numerical results validated against averaged data.

The problem is assumed to be axi-symmetric in order to reduce the size of the CFD model. Effects from e.g. turbulence and buoyancy are clearly three-dimensional by nature. To fully evaluate these effects, three-dimensional CFD calculations should be performed together with experiments that can validate such results. 


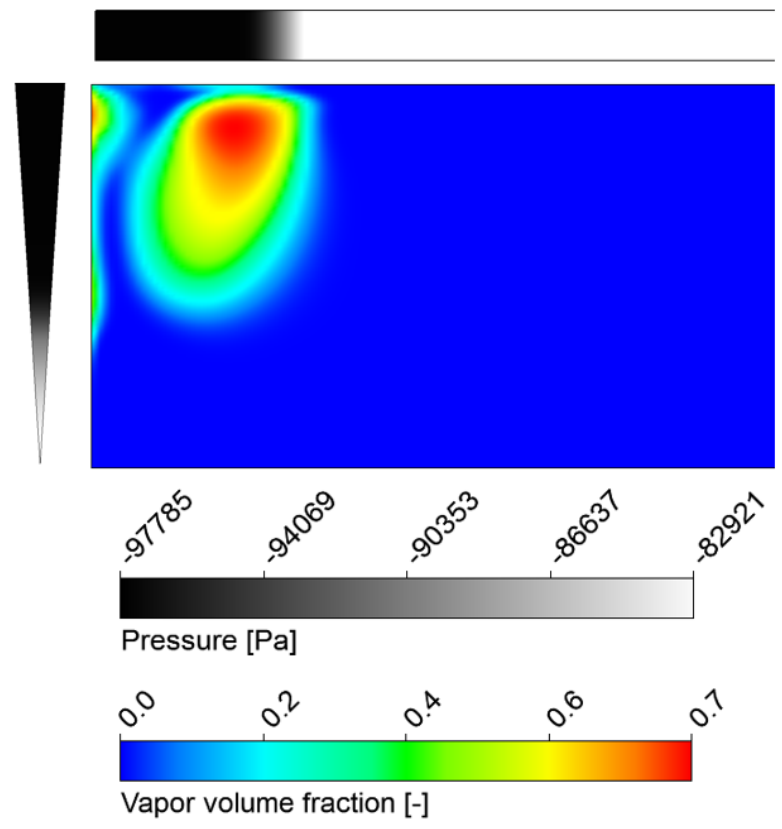

Figure 6: Vapor volume fraction inside the pipe and pressure distribution at the pipe walls.

\section{Conclusions}

In this study, CFD shows great potential for evaluation of hydraulic transients in pipes and exceeds the 1D methods for cavitation flow. Large amplitude hydraulic transients that result in cavitation bubble growth and collapse seem to be well predicted by the CFD model. The effects of cavitation collapse are not seen in the one-dimensional simulations.

To further validate numerical results, experiments that measure both the distribution of cavitation bubbles and the amount of cavitation erosion is necessary.

\section{References}

[1] T. Koivula. On cavitation in fluid power. In Proceedings of 1st FPNI-PhD Symposium, pages 371-382, Hamburg, 2000.

[2] N. Hedlund. Experimental simulation of cavitation erosion. Master's thesis, KTH Royal Institute of Technology, 2014.

[3] J.-P. Franc and J.-M. Michel, editors. Fundamentals of Cavitation. Springer Science \& Business Media, 2010.

[4] C. E. Brennen. Cavitation and Bubble Dynamics. Oxford University Press, 1995.

[5] C. E. Brennen. An introduction to cavitation fundamentals. In WIMRC FORUM 2011 - Cavitation: Turbomachinery \& Medical Applications, University of Warwick, UK, 4-6 July 2011.

[6] K. H. Kim, G. Chahine, J.-P. Franc, and A. Karimi, editors. Advanced Experimental and Numerical Techniques for Cavitation Erosion Prediction. Springer, 2014.
[7] M. H. Chaudhry. Applied Hydraulic Transients. Springer, 2013.

[8] M. R. Nikpour, A. H. Nazemi, A. Hosseinzadeh Dalir, F. Shoja, and P. Varjavand. Experimental and numerical simulation of water hammer. Arabian Journal for Science and Engineering, 39(4):2669-2675, 2014.

[9] F.J. Salvador, J. Martínez-López, M. Caballer, and C. De Alfonso. Study of the influence of the needle lift on the internal flow and cavitation phenomenon in diesel injector nozzles by CFD using RANS methods. Energy Conversion and Management, 66:246-256, feb 2013.

[10] F. Brusiani, S. Falfari, and G. M. Bianchi. Definition of a CFD multiphase simulation strategy to allow a first evaluation of the cavitation erosion risk inside high-pressure injector. Energy Procedia, 81:755-764, dec 2015.

[11] S. I. Bernad and R. Susan-Resiga. Numerical model for cavitational flow in hydraulic poppet valves. Modelling and Simulation in Engineering, 2012:1-10, 2012.

[12] R. Amirante, E. Distaso, and P. Tamburrano. Experimental and numerical analysis of cavitation in hydraulic proportional directional valves. Energy Conversion and Management, 87:208-219, 2014.

[13] A. Peters, H. Sagar, U. Lantermann, and O. el Moctar. Numerical modelling and prediction of cavitation erosion. Wear, 338-339:189-201, sep 2015.

[14] T.-H. Shih, W. W. Liou, A. Shabbir, Z. Yang, and J. Zhu. A new $k-\varepsilon$ eddy-viscosity model for high reynolds number turbulent flows - model development and validation. Computers Fluids, 24(3):227-238, 1995.

[15] G. H. Shnerr and J. Sauer. Physical and numerical modeling of unsteady cavitation dynamics. In Fourth International Conference on Multiphase Flow, New Orleans, USA, 2001.

\section{Nomenclature}

\begin{tabular}{lll}
\hline Designation & Denotation & Unit \\
\hline$n$ & Bubble number density & - \\
$P$ & Far-field pressure & $\mathrm{Pa}$ \\
$P_{v}$ & Vapor pressure & $\mathrm{Pa}$ \\
$R_{B}$ & Bubble radius & $\mathrm{m}$ \\
$R_{c}$ & Condensation mass transfer rate & $\mathrm{kg} /\left(\mathrm{m}^{3} \mathrm{~s}\right)$ \\
$R_{e}$ & Evaporation mass transfer rate & $\mathrm{kg} /\left(\mathrm{m}^{3} \mathrm{~s}\right)$ \\
$\alpha$ & Vapor volume fraction & - \\
$\rho$ & Mixture density & $\mathrm{kg} / \mathrm{m}^{3}$ \\
$\rho_{l}$ & Liquid density & $\mathrm{kg} / \mathrm{m}^{3}$ \\
$\rho_{v}$ & Vapor density & $\mathrm{kg} / \mathrm{m}^{3}$ \\
\hline
\end{tabular}

